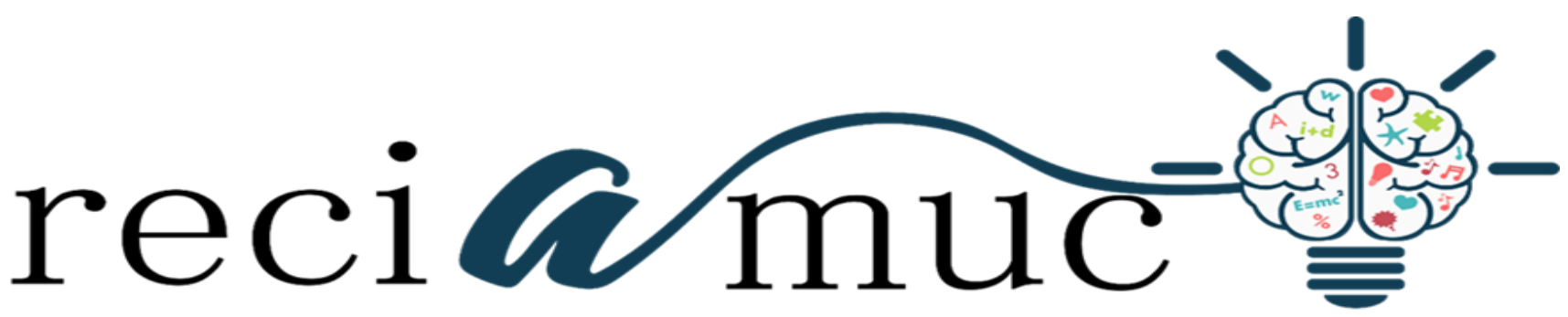

Revista cientifica de investigación actualización del mundo de las ciencias

Daniel Fernando Dick Paredes ${ }^{\text {a; }}$ Jenniffer Cristina Pazmiño Farfán ${ }^{\text {b; }}$ John Michael

Franco Orellana ${ }^{c}$; Adrián André Bravo Legarda ${ }^{\mathrm{d}}$

Trastorno disfórico premenstrual

Premenstrual dysphoric disorder

Revista Científica de Investigación actualización del mundo de las Ciencias. Vol. 3 núm., 1, enero, ISSN: 2588-0748, 2018, pp. 199-217

DOI: $10.26820 /$ reciamuc/3.(1).enero.2019.199-217

URL: http://reciamuc.com/index.php/RECIAMUC/article/view/231

Código UNESCO: 3205 Medicina Interna

Tipo de Investigación: Artículo de Revisión

Editorial Saberes del Conocimiento

Recibido: 10/12/2018

Aceptado: 20/01/2019

Publicado: 30/01/2019

Correspondencia: director@ reciamuc.com

a. Médico; Guayaquil, Ecuador.
b. Médico; Guayaquil, Ecuador.
c. Médico; Guayaquil, Ecuador.
d. Médico; Guayaquil, Ecuador. 


\section{Trastorno disfórico premenstrual}

Vol. 3, núm. 1., (2019)

Alexandra Elizabeth Cedeño Franco; Silvia Gianella Solórzano García; Gema Gabriela Barrezueta Tumbaco, Ruth María Giles Zambrano

\section{RESUMEN}

El desorden disfórico premenstrual es la exacerbación patológica de los cambios somáticos y psíquicos que ocurren durante la fase luteínica en el ciclo femenino normal, ocasiona problemas de relación familiar, laboral y social debido a incapacidad física y psíquica. El síndrome premenstrual es una condición común en la mayoría de la mujeres, entre los 20 a 40 años de edad, que manifiesta síntomas emocionales, físicos y de comportamiento, 5 o 10 días previos al inicio del período menstrual, desapareciendo cuando el período comienza o en el plazo de 4 a 7 días. No tiene una causa exacta que lo genere, algunos estudios revelan que estaría relacionado con cambios en niveles hormonales durante el ciclo menstrual. El diagnóstico se realiza basándose en la medición subjetiva de síntomas que la paciente en un diario o lista anota durante cada día del ciclo menstrual, precisando la aparición o agravamiento de síntomas en período luteal. Algunos síntomas que deben ser anotados: tensión nerviosa, alteraciones del humor, irritabilidad, ansiedad, depresión, llanto, olvidos, insomnio, aumento de las siestas, fatiga, dolor de mamas, migrañas, ansias de comer salado y dulce, problemas de piel, fluctuaciones en el peso, dolores generales. . Los casos leves responden a medidas higiénico - dietéticas como tratamiento (cambios de estilo de vida, límites a la ingesta de carbohidratos, ejercicios aeróbico, evitar el estrés y grupos de apoyo). Los casos con síntomas moderados a graves responden a la prescripción de psicofármacos, antidepresivos, hormonas que tienen como propósito la supresión de la ovulación. La metodología usada es descriptiva, con un enfoque documental, es decir, revisar fuentes disponibles en la red, como google académico, con contenido oportuno y relevante desde el punto de vista científico.

Palabras Claves: Síndrome Premenstrual; Trastorno Disfórico; Período Luteal; Síntomas Somáticos; Síntomas Psiquiátricos; Medidas Higiénico; Dietéticas. 


\title{
Trastorno disfórico premenstrual
}

Vol. 3, núm. 1., (2019)

Daniel Fernando Dick Paredes, Jenniffer Cristina Pazmiño Farfán, John Michael Franco

Orellana, Adrián André Bravo Legarda

\begin{abstract}
Premenstrual dysphoric disorder is the pathological exacerbation of the somatic and psychic changes that occur during the luteal phase in the normal female cycle, causing problems of family, work and social relationship due to physical and psychic disability. Premenstrual syndromeis a common condition in most women, between 20 and 40 years of age, which manifests emotional, physical and behavioral symptoms, 5 or 10 days before the beginning of the menstrual period, disappearing when the period begins or within 4 to 7 days. It does not have an exact cause that generates it, some studies reveal that it would be related to changes in hormonal levels during the menstrual cycle. The diagnosis is made based on the subjective measurement of symptoms that the patient in a diary or list notes during each day of the menstrual cycle, specifying the appearance or aggravation of symptoms in the luteal period. Some symptoms that should be noted: nervous tension, mood changes, irritability, anxiety, depression, crying, forgetfulness, insomnia, increased naps, fatigue, breast pain, migraines, cravings for salty and sweet, skin problems, fluctuations in weight, general pains. . Mild cases respond to hygienic dietary measures such as treatment (lifestyle changes, limits on carbohydrate intake, aerobic exercise, avoiding stress and support groups). Cases with moderate to severe symptoms respond to the prescription of psychotropic drugs, antidepressants, hormones that have the purpose of suppressing ovulation. The methodology used is descriptive, with a documentary approach, that is, to review sources available on the web, such as academic google, with timely and relevant content from the scientific point of view.
\end{abstract}

Key Words: Premenstrual Syndrome; Dysphoric Disorder; Luteal Period; Somatic Symptoms; Psychiatric Symptoms; Hygienic; Dietetic Measures. 


\section{Trastorno disfórico premenstrual}

Vol. 3, núm. 1., (2019)

Alexandra Elizabeth Cedeño Franco; Silvia Gianella Solórzano García; Gema Gabriela Barrezueta Tumbaco, Ruth María Giles Zambrano

\section{Introducción.}

El síndrome premenstrual es una condición común en la mayoría de la mujeres, entre los 20 a 40 años de edad, que manifiesta síntomas emocionales, físicos y de comportamiento, 5 o 10 días previos al inicio del período menstrual, desapareciendo cuando el período comienza o en el plazo de 4 a 7 días. Por el contrario, la exaltación exacerbada patológica de los cambios somáticos y psíquicos que ocurren durante la fase luteínica en el ciclo femenino normal se denomina Trastorno disfórico premenstrual.

En principio son muy parecidos pero los padecimientos del síndrome disfórico son más profundos debido a que afectan la calidad de vida de la mujer que lo sufre. Los cambios físicos y psicológicos comprometen la calidad y productividad de quien la padece, no hay causas específicas a quien atribuirle esta condición, incluso el diagnóstico es complicado para una mujer con bajo nivel instructivo, requiere un trabajo conjunto entre médico y paciente, anotaciones de síntomas en fechas precisas y descartar otro tipo de patologías.

El propósito del presente artículo es crear conciencia de esta realidad que padecen un número de mujeres que representan entre un $5-8 \%$ de la población pero con una inexistente calidad de vida. Son padecimientos severos de los cuales no tiene control quien la sufre. (Bustos, 2018).

Los tratamientos y diagnostico se presentaran a continuación, buscar soluciones o maneras de aliviar a quien la padece, explicando las posibles alternativas que van desde algunas muy económicas como el ejercicio físico diario hasta fármacos específicos para trastornos hormonales y psicológicos. 


\section{Trastorno disfórico premenstrual}

Vol. 3, núm. 1., (2019)

Daniel Fernando Dick Paredes, Jenniffer Cristina Pazmiño Farfán, John Michael Franco

Orellana, Adrián André Bravo Legarda

\section{Metodología.}

Esta investigación está dirigida al estudio del Trastorno disfórico premenstrual, diagnostico, causas y tratamientos. Para realizarlo se usó una metodología descriptiva, con un enfoque documental, es decir, revisar fuentes disponibles en la red, como google académico, con contenido oportuno y relevante desde el punto de vista científico para dar respuesta a lo tratado en el presente artículo y que sirvan de inspiración para realizar otros proyectos. Las mismas pueden ser estudiadas al final, en la bibliografía.

\section{Resultados.}

El desorden disfórico premenstrual es la exacerbación patológica de los cambios somáticos y psíquicos que ocurren durante la fase luteínica en el ciclo femenino normal, ocasiona problemas de relación familiar, laboral y social debido a incapacidad física y psíquica. También es definido como un conjunto de síntomas emocionales, comportamentales y somáticos que se presentan al final de la fase luteínica cuyo desenlace se soluciona con la menstruación. (E. Covini, 2013, pág. 210).

Al describir este desorden o trastorno es conveniente explicar los síntomas en tres aspectos:

- Emocional: los síntomas más comunes son depresión, irritabilidad, tensión, llanto, mayor sensibilidad, alternansa entre tristeza e ira.

- Físico: calambres abdominales, fatiga, inflamación, acné, aumento de peso y retención de líquido. 


\section{Trastorno disfórico premenstrual}

Vol. 3, núm. 1., (2019)

Alexandra Elizabeth Cedeño Franco; Silvia Gianella Solórzano García; Gema Gabriela Barrezueta Tumbaco, Ruth María Giles Zambrano

- Conductual: disminución de la concentración, aislamiento social, disminución de la motivación, atracones de comida, entre otros.

Es importante delimitar que el desorden disfórico premenstrual o disforia de la fase luteínica es diferente de la tensión premenstrual que no es patológica y es frecuente en mujeres ciclantes. Esta delimitación permite abordar las alteraciones premenstruales para no confundir variables fisiológicas normales con patología ciclante. El síndrome premenstrual padecido por el $75-80 \%$ de las mujeres, de sintomatología predominantemente somática y no incapacitante versus el desorden disfórico premestrual padecido por el $5-8 \%$ de las mujeres con sintomatología somática y afectiva (trastornos del ánimo) que generan disfunción o incapacidad significativa. A continuación se presenta un cuadro que resume esta comparación de síndromes. (Borrás, 2001).

Tabla $N^{\circ} 1$. Desorden disfórico vs Síndrome premenstrual

VARIABLE

POBLACIÓN QUE

PADECE ESTA CONDICIÓN SÍNTOMAS SOMÁTICOS

SÍNTOMAS PSIQUIÁTRICOS
DESORDEN DISFÓRICO

$$
5-8 \%
$$

Mastodinia.

Edemas.

Hiperorexia.

Irritabilidad.

Labilidad emocional.

Depresión.

Ansiedad.

Insomnio o hipersomnia.

Falta de energía.

Disminución del rendimiento intelectual.

Incapacidad y/o disfunción significativa.
SÍNDROME PREMENSTRUAL

$75-80 \%$

Mastalgia.

Edemas.

Incapacidad leve o ausente.

(n)




\section{Trastorno disfórico premenstrual}

Vol. 3, núm. 1., (2019)

Daniel Fernando Dick Paredes, Jenniffer Cristina Pazmiño Farfán, John Michael Franco

Orellana, Adrián André Bravo Legarda

Se ha determinado que el pico de máxima elevación sintomática se produce en la cuarta década de la vida, iniciándose en la segunda década, no comienza con la menarca o primeras menstruaciones. Las pacientes describen sus síntomas como una banda que articula al cuerpo entero, los movimientos se vuelven más lentos, las extremidades más pesadas y frías, la paciente se angustia, no se siente cómoda, sus ritmos biológicos están alterados, predominando la hipersomnia, evacuación intestinal lenta o ausente, falta de apetito acompañada por antojos peculiares. El chocolate suele ser el más antojado, siendo infrecuente buscar alimentos fuera de la ingesta normal o rutinaria. (Márquez, 2013, pág. 37)

Factores de riesgo asociados al Trastorno Disfórico premenstrual (Bocchino, 2004).

- Se presenta frecuentemente entre los 25 - 35 años de edad.

- Duración atípica de los ciclos menstruales, reportándose mayor incidencia en mujeres con ciclos más largos o más cortos que los promedios o normales.

- Los anticonceptivos orales no inciden en su aparición.

- Las mujeres con trastornos psiquiátricos pasados o actuales son más propensas a padecer trastorno disfórico premenstrual.

- Historia familiar de síndrome premenstrual, se considera una entidad hereditaria.

- Estresores psicosociales como el abuso físico o sexual. Mujeres con antecedentes de abuso tenían niveles de noradrenalina en reposo significativamente más bajo y mayor respuesta adrenérgica al estrés en la fase lútea. Se encontró aplanamiento de la curva de cortisol y alteraciones en el rendimiento cardíaco con relación a la respuesta al estrés en mujeres con antecedentes de abuso. 


\section{Trastorno disfórico premenstrual}

Vol. 3, núm. 1., (2019)

Alexandra Elizabeth Cedeño Franco; Silvia Gianella Solórzano García; Gema Gabriela Barrezueta Tumbaco, Ruth María Giles Zambrano

¿Qué causa el trastorno disfórico premenstrual?

No tiene una causa exacta que lo genere, algunos estudios revelan que estaría relacionado con cambios en niveles hormonales durante el ciclo menstrual. El nivel de sustancias químicas en el cerebro o neurotransmisores como la serotonina puede contribuir al síndrome premenstrual. Otras investigaciones apuntan a la existencia de un gen heredado por algunas mujeres que favorece el desarrollo del trastorno disfórico. Si una mujer tiene estos síntomas y no se detienen al poco tiempo de inicio de su período menstrual, es posible que tenga otro trastorno como depresión.

\section{Diagnóstico}

Tanto el síndrome premenstrual como el trastorno disfórico no se diagnostican con análisis de sangre u otras pruebas de laboratorio. Los doctores identifican los síntomas físicos y emocionales de la mujer durante dos o tres ciclos menstruales y el momento que se manifiestan con relación al período. En el trastorno disfórico deben presentarse al menos cinco síntomas emocionales durante la mayoría de los ciclos en un año, ocasionando la interferencia de actividades o relaciones cotidianas, las cuales mejoran pocos días del inicio del período.

Este diagnóstico se realiza basándose en la medición subjetiva de síntomas que la paciente en un diario o lista anota durante cada día del ciclo menstrual, precisando la aparición o agravamiento de síntomas en período luteal. Algunos síntomas que deben ser anotados: tensión nerviosa, alteraciones del humor, irritabilidad, ansiedad, depresión, llanto, olvidos, insomnio, aumento de las siestas, fatiga, dolor de mamas, migrañas, ansias de comer salado y dulce, problemas de piel, fluctuaciones en el peso, dolores generales. 


\section{Trastorno disfórico premenstrual}

Vol. 3, núm. 1., (2019)

Daniel Fernando Dick Paredes, Jenniffer Cristina Pazmiño Farfán, John Michael Franco

Orellana, Adrián André Bravo Legarda

Los criterios de diagnósticos propuestos por la siguiente clasificación propuesta en el Manual Diagnóstico y Estadístico de los Trastornos Mentales (DSM IV TR, año 2002) (Bocchino, 2004, pág. 79) :

A. Cinco o más de los síntomas durante la mayor parte del día de la última semana de la fase lútea de la mayoría de los ciclos menstruales del último año, que inician dos días después del comienzo de la fase folicular y desaparecen por completo a la semana siguiente a la menstruación:

- Estado de ánimo deprimido, sentimiento de desesperanza e ideas de desaprobación.

- Ansiedad, tensión, sensación de agobio o de estar al límite.

- Labilidad emocional evidente.

- Enfado, irritabilidad o aumento de conflictos interpersonales de forma acusada y persistente.

- Perdida de interés por las actividades cotidianas, es decir, trabajo, amigos, aficiones.

- Sensación subjetiva de dificultad para concentrarse.

- Letargia, fatigabilidad fácil o falta evidente de energía.

- Cambios significativos del apetito, exceso de comida o antojos por determinados alimentos.

- Hipersomnia o insomnio.

- Sensación subjetiva de estar rebasada o fuera de control. 


\section{Trastorno disfórico premenstrual}

Vol. 3, núm. 1., (2019)

Alexandra Elizabeth Cedeño Franco; Silvia Gianella Solórzano García; Gema Gabriela Barrezueta Tumbaco, Ruth María Giles Zambrano

- Otros síntomas físicos como hipersensibilidad o aumento del tamaño mamario, dolores de cabeza, molestia articulares o musculares, sensación de hinchazón o ganancia de peso.

B. Las alteraciones mencionadas anteriormente, interfieren marcadamente con la rutina diaria de la paciente en el trabajo, escuela, actividades sociales, disminución de la productividad y eficiencia en el ámbito laboral o académico.

C. La alteración no representa una simple elevación de síntomas de otros trastornos.

D. Los criterios A, B y C deben ser validados por técnicas de seguimiento diario con énfasis en los síntomas en al menos dos ciclos sintomáticos consecutivos.

La mayor dificultad que presenta el esquema propuesto anteriormente consiste en la poca confiabilidad de los valores diarios y evaluación de los síntomas en pacientes que presenten alguna dificultad en su rendimiento intelectual.

Es importante diferenciar clínicamente el síndrome disfórico premenstrual de otras enfermedades psiquiátricas:

- Depresión: la evaluación prospectiva de los síntomas en la fase folicular y luteínica, permite diferenciar los síntomas afectivos premenstruales de los síntomas crónicos que se presentan en episodios depresivos mayor.

- Trastorno de ansiedad: elevado nivel de ansiedad coexistente con ataques de pánicos en la fase premenstrual, puede observarse comportamientos obsesivos compulsivos. 


\section{Trastorno disfórico premenstrual}

Vol. 3, núm. 1., (2019)

Daniel Fernando Dick Paredes, Jenniffer Cristina Pazmiño Farfán, John Michael Franco

Orellana, Adrián André Bravo Legarda

- Trastorno afectivo bipolar: se ha observado exacerbación de síntomas bipolares en la etapa premenstrual.

- Trastornos de alimentación: el consumo y apetito por los carbohidratos se eleva en la época premenstrual y se asocia con estados de humor depresivo en mujeres sanas. En mujeres con desorden de alimentación es difícil de discriminar si los síntomas son por trastorno disfórico o alimenticio.

- Abuso de sustancias psicoactivas: el consumo de alcohol en la etapa premenstrual parece estar más asociado a un historial familiar de alcoholismo, drogas o nicotina que a un trastorno disfórico premenstrual.

- Condiciones médicas generales: varias enfermedades como endometriosis, desordenes tiroideos, trastorno del sistema adrenal, hiperprolactinemia y panhipopituarismo se asemejan en algunos síntomas del trastorno disfórico, hay que descartarlas durante el diagnostico.

Factores etiopatogéncos

Los diferentes hallazgos que evidencian la multiplicidad de factores involucrados se encuentran resumidos en la siguiente tabla: 


\section{Trastorno disfórico premenstrual}

Vol. 3, núm. 1., (2019)

Alexandra Elizabeth Cedeño Franco; Silvia Gianella Solórzano García; Gema Gabriela Barrezueta Tumbaco, Ruth María Giles Zambrano

\section{Tabla $N^{\circ}$ 2. Factores Etiopatogénicos}

\begin{tabular}{|c|c|}
\hline Factores genéticos & Factores hormonales \\
\hline Concordancia en gemelos e historia & Secreción de prolactina. \\
familiar. Antecedentes de PMDD. & Secreción de GH. \\
Alteraciones en los sistemas de & Eje tiroideo. \\
neurotransmisión. & Secreción de cortisol basal. \\
Disminución de la actividad de la MAO & Secreción de melatonina. \\
por acción de la progesterona. & Secreción de PGE y PGF. \\
Aumento de la actividad NA. & \\
Disminución del tono 5-HT por \\
esteroideos sexuales. \\
Deficiencia de progesterona. \\
Alteración en relación \\
estrógenos/progesterona. \\
Alteración de la secreción de \\
testosteronas.
\end{tabular}

Fuente: Márquez Andrea, 2013

\section{Tratamiento}

El amplio número de síntomas, modalidades de aparición e intensidad de los síntomas del trastorno disfórico, amerita una observación y seguimiento del paciente que oriente la elección terapéutica más oportuna. Los casos leves responden a medidas higiénico - dietéticas (cambios de estilo de vida, límites a la ingesta de carbohidratos, ejercicios aeróbico, evitar el estrés y grupos de apoyo). Algunos cambios propuestos para estas pacientes:

- Comer comidas pequeñas y frecuentes.

- Reducir la ingesta de sal.

- Optar por verduras y granos enteros y alimentos con un alto contenido de proteína.

- Realizar ejercicio diario por 30 minutos al día, la mayorías de los días de la semana. 


\section{Trastorno disfórico premenstrual}

Vol. 3, núm. 1., (2019)

Daniel Fernando Dick Paredes, Jenniffer Cristina Pazmiño Farfán, John Michael Franco

Orellana, Adrián André Bravo Legarda

- Reducir el estrés.

- Dormir lo suficiente.

- No fumar y disminuir el consumo de cafeína, chocolate y alcohol.

El soporte psicoterapéutico, resulta beneficiosos en algunas pacientes porque permite a las mujeres conocer más de cerca sus síntomas, el ritmo de aparición, sus desencadenantes y exacerbaciones. El monitoreo y seguimiento de los síntomas permite desarrollar conciencia en las pacientes, facilitando cambios en el estilo de vida cuyo premio es prevenir agudizaciones o recaídas. Los ejercicios de relajación como el yoga, ejercicios focalizados al manejo del estrés y aprendizaje de habilidades son muy oportunos.

Los casos leves a moderados requieren además de los ya sugeridos, tratamientos con suplementos vitamínicos y minerales como:

- El consumo de calcio $1200 \mathrm{mg}$ diarios junto a las vitaminas D, E $400 \mathrm{mg}$ y B6 100 mg y magnesio $200 \mathrm{mg}$ por día.

Otros productos que alivian este padecimiento son productos herbales (disminuyen los síntomas pero existe poco conocimiento de dosis, eficacia y seguridad en su aplicación). El vitex agnus o planta sauzgatillo mejora la irritabilidad, disforia y la mastalgia. El dong quai o ginseng femenino mejora los dolores y ciclo menstrual. La valeriana se usa para el tratamiento de la ansiedad y el insomnio, mejora la digestión, es diurética. El aceite de prímula con altos índices de ácido linoleico y omega 6, alivia la disforia, la mastalgia, la dismenorrea, mejora la acción inmunológica, disminuye la exacerbación luteal de enfermedad autoinmune. 


\section{Trastorno disfórico premenstrual}

Vol. 3, núm. 1., (2019)

Alexandra Elizabeth Cedeño Franco; Silvia Gianella Solórzano García; Gema Gabriela Barrezueta Tumbaco, Ruth María Giles Zambrano

Los casos con síntomas moderados a graves responden a la prescripción de psicofármacos, antidepresivos, hormonas que tienen como propósito la supresión de la ovulación. Se escriben los más conocidos:

- El consumo de diuréticos ayuda a aliviar la hinchazón.

- Analgésicos como ibuprofeno o naproxeno.

- Pastillas anticonceptivas.

- Agonistas de la hormona liberadora de gonadotropina.

- Antidepresivos parecen ser hasta ahora la estrategia más efectiva para este trastorno.

- Medicamentos contra la ansiedad como alprazolam, su eficacia es menor que los antidepresivos.

En un estudio propuesto por Steiner para 313 pacientes, administra $20 \mathrm{mg} /$ día de fluoxetina, $60 \mathrm{mg} /$ día fluoxetina y placebo, todas fueron evaluadas con escala Visual AnalogScale que cuantifica la tensión emocional, irritabilidad y la distrofia. Los resultados fueron una mejoría con ambas dosis pero menor en el grupo que recibió 20 mg/día. Estos resultados avalan la efectividad sobre la sintomatología psicológica y física, así como el rápido inicio de acción. (Márquez, 2013, pág. 41)

Para el edema y la retención de líquidos se prescribe la espironolactona, mejora la irritabilidad, la depresión, sensación de inflamación, sensibilidad mamaria y el deseo de comer. La dosis recomendada es de $100 \mathrm{mg}$ dividida en dos a cuatro tomas desde el día 14 hasta la menstruación. El uso efectivo de cualquier fármaco puede verse afectado si la paciente ingiere anticonceptivos orales debido a que ellos contienen altas dosis de estrógenos. 


\section{Trastorno disfórico premenstrual}

Vol. 3, núm. 1., (2019)

Daniel Fernando Dick Paredes, Jenniffer Cristina Pazmiño Farfán, John Michael Franco

Orellana, Adrián André Bravo Legarda

Algunas cifras

Un trabajo de investigación descriptivo realizado en la Facultad de Medicina de la Universidad Concepción en Chile, con una muestra el azar de 340 mujeres con edades comprendidas entre los 18 - 27 años de edad se encontró lo siguiente (Bustos, 2018):

De las estudiantes encuestadas se pudo conocer que el 55.9\% presenta síntomas relacionados al síndrome del trastorno premenstrual, número más alto en relación al obtenido en el año 2014 para la misma población $47.8 \%$ pero inferior al $66 \%$ obtenido en un estudio de prevalencia del trastorno premenstrual realizado en Ecuador en el año 2011 en una muestra con características similares. Estos estudios son importantes porque miden el impacto de este síndrome en la vida de la mujer, si persiste a lo largo del tiempo puede afectar no sólo su bienestar físico sino también social y emocional.

En el año 2012 una revisión efectuada por Besio, se describen los síntomas manifestados por adolescentes tanto chilenas como argentinas, cuyos resultados coinciden con los de ese estudio: fatiga y falta de energía (64.7\%), hipersensibilidad mamaria, cefalea e hinchazón (62.9\%) y ansiedad, tensión, agobio y colapso (60.9\%) lo que se corresponde con los hallazgos de otros estudios efectuados en Argentina 18\% y en Brasil 20\%.

\section{Conclusiones.}

El trastorno disfórico premenstrual es definido como un conjunto de síntomas emocionales, comportamentales y somáticos que se presentan al final de la fase luteínica cuyo 


\section{Trastorno disfórico premenstrual}

Vol. 3, núm. 1., (2019)

Alexandra Elizabeth Cedeño Franco; Silvia Gianella Solórzano García; Gema Gabriela Barrezueta Tumbaco, Ruth María Giles Zambrano

desenlace se soluciona con la menstruación pero se repite en cada período y se agudiza en la cuarta década.

Al describir este desorden o trastorno es conveniente explicar los síntomas en tres aspectos:

- Emocional: los síntomas más comunes son depresión, irritabilidad, tensión, llanto, mayor sensibilidad, alternansa entre tristeza e ira.

- Físico: calambres abdominales, fatiga, inflamación, acné, aumento de peso y retención de líquido.

- Conductual: disminución de la concentración, aislamiento social, disminución de la motivación, atracones de comida, entre otros.

No tiene una causa exacta que lo genere, algunos estudios revelan que estaría relacionado con cambios en niveles hormonales durante el ciclo menstrual. El nivel de sustancias químicas en el cerebro o neurotransmisores como la serotonina puede contribuir al síndrome premenstrual. Otras investigaciones apuntan a la existencia de un gen heredado por algunas mujeres que favorece el desarrollo del trastorno disfórico.

Los doctores identifican los síntomas físicos y emocionales de la mujer durante dos o tres ciclos menstruales y el momento que se manifiestan con relación al período. En el trastorno disfórico deben presentarse al menos cinco síntomas emocionales durante la mayoría de los ciclos en un año, ocasionando la interferencia de actividades o relaciones cotidianas, las cuales mejoran pocos días del inicio del período. Los criterios de diagnósticos propuestos por la siguiente clasificación propuesta en el Manual Diagnóstico y Estadístico de los Trastornos Mentales (DSM IV TR, año 2002). 


\section{Trastorno disfórico premenstrual}

Vol. 3, núm. 1., (2019)

Daniel Fernando Dick Paredes, Jenniffer Cristina Pazmiño Farfán, John Michael Franco

Orellana, Adrián André Bravo Legarda

Cinco o más de los síntomas durante la mayor parte del día de la última semana de la fase lútea de la mayoría de los ciclos menstruales del último año, que inician dos días después del comienzo de la fase folicular y desaparecen por completo a la semana siguiente a la menstruación:

- Estado de ánimo deprimido, sentimiento de desesperanza e ideas de desaprobación.

- Ansiedad, tensión, sensación de agobio o de estar al límite.

- Labilidad emocional evidente.

- Sensación subjetiva de dificultad para concentrarse.

- Letargia, fatigabilidad fácil o falta evidente de energía.

- Cambios significativos del apetito, exceso de comida o antojos por determinados alimentos.

- Hipersomnia o insomnio.

Los tratamiento abarcan una amplia gamaen atención al número de síntomas, modalidades de aparición e intensidad de los síntomas del trastorno disfórico, amerita una observación y seguimiento del paciente que oriente la elección terapéutica más oportuna. Los casos leves responden a medidas higiénico - dietéticas (cambios de estilo de vida, límites a la ingesta de carbohidratos, ejercicios aeróbico, evitar el estrés y grupos de apoyo). Algunos cambios propuestos para estas pacientes:

- Comer comidas pequeñas y frecuentes.

- Reducir la ingesta de sal.

- Realizar ejercicio diario por 30 minutos al día, la mayorías de los días de la semana. 


\section{Trastorno disfórico premenstrual}

Vol. 3, núm. 1., (2019)

Alexandra Elizabeth Cedeño Franco; Silvia Gianella Solórzano García; Gema Gabriela Barrezueta Tumbaco, Ruth María Giles Zambrano

- No fumar y disminuir el consumo de cafeína, chocolate y alcohol.

Los casos leves a moderados requieren además de los ya sugeridos, tratamientos con suplementos vitamínicos y minerales como: el consumo de calcio, junto a las vitaminas D, E y magnesio. Los casos con síntomas moderados a graves responden a la prescripción de psicofármacos, antidepresivos, hormonas que tienen como propósito la supresión de la ovulación. Para el edema y la retención de líquidos se prescribe la espironolactona, mejora la irritabilidad, la depresión, sensación de inflamación, sensibilidad mamaria y el deseo de comer. (López, 2000).

\section{Bibliografía.}

Bocchino, S. (2004). Salud mental de la mujer. Síntomas y trastornos premestruales. Clínica y tratamiento. Revista de psiquiatría del Uruguay volumen $68 N^{\circ} 1,78$ - 89.

Borrás, C. (2001). Ansiedad y diagnóstico del sindrome premestrual. Red de revistas cientificas de América Latina y el Caribe, España y Portugal, volumen $12 N^{\circ} 3,341$ - 366.

Bustos, R. (2018). Influencia de la actividad física en la sintomatología del síndrome premestrual en un grupo de mujeres de la Facultad de Medicina de la Universidad de Concepción. Revista Chilena de Obstetricia y Ginecología, volumen $83 N^{\circ} 1,45$ - 51.

E. Covini, A. M. (2013). Trastorno disfórico premenstrual o PMDD. Alcmeon, Revista Argentina de Clínica Neuropsiquiatrica, 201-221.

Fundación de Hormonas. (2010). Sindrome premestrual y trastorno disfórico premestrual. The Journal of clinical endocrinilogia \& metabolism, 2.

López, A. (2000). Trastorno disfórico premestrual. Revista Chilena de Neuropsiquiatria, volumen $38 N^{\circ} 3,187$ - 195.

Márquez, A. (2013). Trastorno disfórico premestrual. Revista de Endocrinología Ginecológica y reproductiva, 36 - 45 . Obtenido de www.saegre.org.ar

Perarnau, M. (2010). Síntomas, síndrome y trastorno disfórico premenstrual en una muestra de mujeres universitarias. Recuperado el 28 de Abril de 2019, de Recuperado por http: www.fundamentos.unsl.edu.ar 


\section{Trastorno disfórico premenstrual}

Vol. 3, núm. 1., (2019)

Daniel Fernando Dick Paredes, Jenniffer Cristina Pazmiño Farfán, John Michael Franco

Orellana, Adrián André Bravo Legarda

Zanin, L. (2012). Ciclo menstrual: sintomatología y regularidad del estilo de vida diario. Recuperado el 28 de Abril de 2019, de Recuperado por http: www.redalyc.org 